\title{
Territorio estratégico y segregación: la región noreste del Área Metropolitana de Montevideo
}

\section{Strategic territory and segregation: the northeast region of the Metropolitan Area of Montevideo}

\author{
Mauricio Ceroni* \\ Aline DA FonseCA* \\ FELINE SCHÖN* \\ Ana Domínguez*
}

\begin{abstract}
Latin American metropolitan areas currently undergo various regional changes which have intensified over the past decades. This study analyzes the territorial dynamics that have occurred in the Northeast Region of the Metropolitan Area of Montevideo, considering concepts such as territorial segregation and strategic territories as a key to understand these changes. Geoprocessing techniques are used and an index of socioeconomic stratification is produced. The results indicate a steady growth of urbanization pressing on rural uses, showing a process of rapid change in different social classes in which the State is one of the key actors to favor territorial segregation.
\end{abstract}

Keywords: territorial segregation, strategic territory, land use, Metropolitan Area of Montevideo.

\section{Resumen}

Las áreas metropolitanas de América Latina transitan por diversos cambios territoriales que se han profundizado durante las últimas décadas. En este estudio se analizan las dinámicas territoriales que han ocurrido en la región noreste del Área Metropolitana de Montevideo, se considera a la segregación territorial y a los territorios estratégicos como conceptos claves para entender dichas transformaciones. En este sentido se emplean técnicas de Geoprocesamiento y se elabora un índice de estratificación socioeconómica. Los resultados indican un crecimiento sostenido de los procesos de urbanización sobre el uso rural y se evidencia un desarrollo acelerado de cambios en las distintas clases sociales donde el Estado es un actor clave para favorecer los procesos de segregación territorial.

Palabras claves: segregación territorial, territorio estratégico, uso de suelo, Área Metropolitana de Montevideo.

* Universidad de la República de Uruguay, Uruguay. Correos-e: mceroni@ei.udelar.edu.uy, adafonseca@pim.edu.uy, fschon@fcien.edu.uy y anitad@fcien.edu.uy 


\section{Introducción}

Dentro del marco de la globalización, América Latina atraviesa una serie de transformaciones urbanas que presentan similitudes y especificidades de acuerdo con el contexto de los países (Veiga, 2013; Hernández, 2009). Dichas transformaciones están comandadas, en muchos casos, por una producción del espacio urbano de carácter neoliberal, que se establece bajo las bases de la materialidad capitalista a través de muchas estrategias como privatizaciones de espacios públicos, fraccionamientos o barrios privados, megaedificios, parques industriales, viviendas de interés social, entre otros (Harvey, 2001).

En el caso de Uruguay, algunas transformaciones urbanas explicitan la presencia de procesos globales neoliberales que influyen en su aceleración. El país presenta características diferentes con respecto a la mayoría de los países latinoamericanos, pues tiene una inserción pasiva ${ }^{1}$ en la economía-mundo capitalista (Falero, 2011), además de una población envejecida y que crece muy lentamente (Cepal, 2012).

Dentro de Uruguay, el Área Metropolitana de Montevideo (AMM) es la de mayor extensión en superficie y presenta características especiales. Según la definición de Veiga (2013) está integrada por "áreas urbanas y territorios que se extienden desde el centro montevideano hasta un radio de aproximadamente 30 kilómetros, hacia los departamentos de Canelones y San José”. Dentro de las particularidades, se aprecia una heterogeneidad espacial acompañada de dinámicas muy diferentes, materializadas en los contrastes del paisaje.

La región noreste del AMM, específicamente la zona de influencia de los ejes viales nacionales No 101 y No 8 , se caracteriza porque presenta un desarrollo particular, principalmente debido a urbanización: ejes viales estratégicos, remodelación y ampliación del aeropuerto internacional, la instalación de zonas francas ${ }^{2}$ (Zonamerica ${ }^{3}$ y Parque de las Ciencias ${ }^{4}$ ) y el desarrollo de grandes barrios privados, por lo que la zona es de gran interés para su análisis debido a la celeridad de los cambios, proceso que no ocurre en otra parte del AMM.

${ }^{1} \mathrm{El}$ autor refiere a la inserción pasiva como una posición dependiente del Uruguay en las dinámicas de acumulación como proveedor de "servicios" y materia prima sin promover otras alternativas sociales y económicas (Falero, 2011).

${ }^{2}$ Dentro del artículo 2 de la Ley 15.921 (de 17 de diciembre de 1987) las zonas francas son áreas del territorio nacional de propiedad pública o privada, cercadas y aisladas eficientemente, con el fin de que se desarrollen en ellas toda clase de actividades industriales, comerciales o de servicios, con exoneraciones tributarias.

${ }^{3}$ Veáse http://web.zonamerica.com/home/

${ }^{4}$ Veáse http://www.parquedelasciencias.net/es/zona-franca.php 
Se parte de la premisa de que sobre el área de estudio incide la lógica de los territorios estratégicos, caracterizada por una serie de factores que ocurren encadenadamente (creación de infraestructura vial, zonas francas y barrios privados, etc.) y que se enmarcan dentro de una lógica más global, evidenciando cómo estos territorios generan procesos de diferenciación y segregación territorial.

Para identificar tales lógicas se emplean técnicas de mapeamiento de usos de suelo y distribución de las clases sociales. El artículo está integrado por cuatro apartados, el primero asociado al marco teórico en el que se definen conceptos claves como segregación territorial y territorios estratégicos. Luego, se especifica la metodología desarrollada exponiendo las técnicas y métodos utilizados, a continuación se enuncian los resultados y su discusión, finalmente, se presentan algunas consideraciones con respecto a la situación actual de ambos grupos (empresarios y propietarios de los terrenos).

\section{Conceptos claves}

Hasta la actualidad son escasos los estudios sobre los aspectos territoriales de esta región del país; los existentes abordan dos amplios enfoques dentro de las ciencias sociales; por un lado, los estudios que consideran los procesos de urbanización a partir del análisis de la estructura, centralidad y morfología urbana (Lombardi y Bervejillo, 1999; Schelotto, 2008), así como la dinámica en la variación de viviendas y población (Martínez, 2011); por otra parte, los que analizan los procesos de desigualdad y su fragmentación social (Falero et al., 2013; Veiga y Rivoir, 2001; Veiga, 2013).

Los estudios sobre zonas francas (Falero, 2011) del AMM han sido más específicos pues se enfocan en barrios privados (Álvarez-Rivadulla, 2007) y matriz productiva (Mendy y González, 2010). Estos autores analizan diferentes fenómenos que ocurren en el AMM e identifican procesos y consecuencias comunes, tales como la desigualdad, la segregación y una intensificación de flujos financieros en muchos casos extranjeros.

Ahora bien, es necesario aclarar ciertos conceptos que posibilitan comprender y dialogar con estas dinámicas, entre ellos la desigualdad social, a partir de la cual se configuran diferentes territorios y se generan distintos tipos de segregación. Ésta es la marca de las sociedades capitalistas, si se tiene en cuenta que cada sociedad presenta procesos históricos específicos, como apunta Bourdieu (1998), la jerarquía está presente en todos sus ámbitos, en diferentes escalas y muchas veces camuflada como natural. La desigualdad es resultado de las relaciones sociales, políticas y culturales, siendo el espacio geográfico su expresión más visible, donde 
se puede observar la configuración de distintos territorios en conflicto (Martins, 1997; Oliveira, 1998).

Una de las formas más visibles de la desigualdad es la segregación, concepto muy utilizado en los estudios urbanos, a partir de diferentes enfoques y metodologías (Pérez-Campuzano y Santos-Cerquera, 2011). Según Veiga y Rivoir (2001) la segregación no es sólo una desigualdad social sino un proceso y un resultado de las diferencias sociales y espaciales. Según García-Gómez y Ruiz-Salazar (2011: 5) es "una combinación de disparidades sociales y geográficas para los diferentes sectores de la población que resultan en una fragmentación que se muestra en condiciones desiguales y calidades de vida diferente que tienen los ciudadanos". La segregación socioespacial es una forma del proceso de la diferenciación social, la cual se muestra en la distribución de la estructura social y sus clases en el espacio, diferenciándose en tres niveles asociados a distintos ámbitos organizacionales: "el nivel colectivo de interacción social, el nivel físico-espacial y el nivel de procesos simbólicos y psicológicos que estructuran las conductas individuales" (Machado Barbosa en Veiga y Rivoir, 2001: 10).

Se entiende que el concepto de la segregación socioespacial en algunos casos puede llevar a confundir los conceptos social y espacial como si fueran dos instancias separadas. Por eso se utilizará el concepto de la segregación territorial que explicita claramente la dialéctica entre las relaciones sociales y el espacio. Las ciencias sociales y especialmente la geografía realizan una profunda reevaluación sobre el concepto de territorio a partir de sus múltiples manifestaciones. Entre los autores que reflexionan sobre esa temática destacan Marcos Aurelio Saquet y Rogério Haesbaert.

Saquet (2007) identifica y caracteriza cuatro perspectivas de enfoque en los estudios del territorio que se realizan en el tiempo histórico y coexisten en algunas situaciones, momentos o periodos: la primera, eminentemente económica, sobre el materialismo histórico y dialéctico, entendiendo al territorio a partir de las relaciones de producción y de las fuerzas productivas; la segunda, pautada bajo la dimensión geopolítica del territorio; la tercera, enfocada hacia las dinámicas políticas, culturales y simbólico-identitarias, tratando de representaciones sociales, centradas en la fenomenología y, la cuarta, que gana fuerza a partir de 1990, asociada a las discusiones sobre la sustentabilidad ambiental y al desarrollo local.

Araújo y Haesbaert (2009) destacan que el territorio se construye en el juego de la materialidad e inmaterialidad, de lo funcional y de lo simbólico, desde una perspectiva que valoriza las relaciones y los procesos (territorio en el sentido relacional y procesual), las múltiples temporalidades y velocidades, las cuales se construyen por medio de la conjugación 
entre un continuo que se extiende desde el más funcional hasta el más simbólico.

Lejos de intentar hacer una revisión de cómo se ha tratado el concepto, se entiende que el territorio es construido social e históricamente, puede tener un carácter material o inmaterial, contiene aspectos identitarios y relaciones de poder, es multidimensional y multiescalar. Así, éste se define con relación a la apropiación y al dominio de un espacio socialmente compartido, donde se concentran las relaciones sociales y de poder. De esa manera, el territorio puede ser pensado por múltiples relaciones, económicas, políticas y culturales.

En el contexto actual, independientemente de cómo es definido (acumulación flexible, globalización, revolución informacional), el territorio se expresa con mayor claridad ante las desigualdades sociales y la propia segregación. Dentro de esa lógica, se encuentran los territorios estratégicos, que como apunta Saskia Sassen (2007) expresan tendencias críticas en la reconfiguración del orden social, donde tanto la ciudad como la región metropolitana constituyen lugares estratégicos para la materialización de ciertas tendencias macrosociales importantes, que se configuran como una nueva geografía de la centralidad.

En este sentido, parte de los fenómenos que hoy caracterizan a Uruguay podrían ser ejemplos de localización de procesos globales (Sassen, 2007). La autora destaca que en las investigaciones se deben tener en cuenta los efectos de configuración de territorios estratégicos del capital en el tejido social: cómo se materializan los procesos globales y los vínculos que conectan la escala global y subnacional y las acciones del Estado en sus distintas escalas (nacional, departamental y local), en la transformación de geografías económicas específicas que se van cristalizando sobre la estructura social.

En esta línea conceptual, se entiende que el área de estudio responde a ciertas lógicas que plantea Sassen, se trata de procesos subnacionales que pueden ser codificados como instancias de lo global, en donde su consecuencia más visible puede verse reflejada por intermedio de la segregación. En este panorama, se busca identificar la distribución de las clases sociales, que servirá como una herramienta analítica para observar procesos de diferenciación, desigualdad social y segregación. Con el fin de comprender mejor las transformaciones y procesos que inciden sobre el área de estudio se generan algunas interrogantes: ¿Cuáles son las principales dinámicas y elementos territoriales en la región noreste del AMM? ¿Cómo se expresan las clases sociales con base en estas dinámicas? 


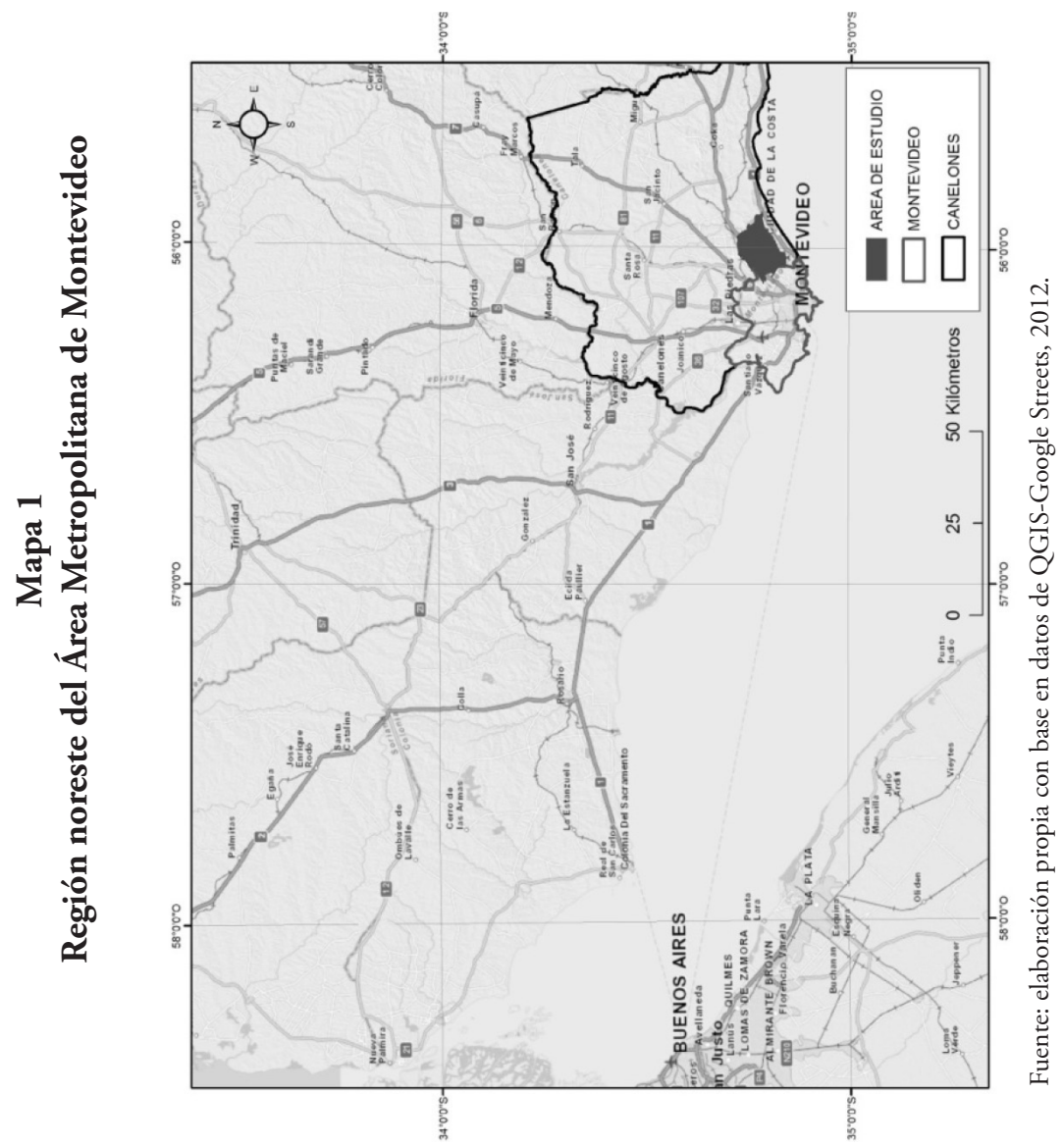




\section{Metodología}

Se identificaron y analizaron las dinámicas territoriales que han ocurrido en el AMM durante el periodo 1993-2013. Cabe explicar que este es un estudio descriptivo-analítico en donde la comprensión de la dinámica territorial se realizó con base en dos métodos: la clasificación de usos de suelo; es decir, a partir del diagnóstico de la funcionalidad principal del uso; y una estratificación socioeconómica basada en datos del censo de población y vivienda del periodo 1996-2011. La elaboración, sistematización y análisis de los datos se realizó mediante la construcción de un Sistema de Información Geográfica (SIG) para el área de estudio, utilizando el programa informático Quantum Gis 2.0.

\section{1. Área de estudio}

El foco de análisis del estudio se centra en la región noreste del AMM que ocupa $13,4 \mathrm{~km}^{2}$ y se localiza entre los límites de los departamentos de Montevideo y Canelones, Barros Blancos y Colonia Nicolich son los centros poblados de mayor importancia (mapa 1); Esta zona se caracteriza por ser el contacto entre la expansión de la mancha urbana y la presencia de zonas rurales, cuyo parcelario es de pequeñas superficies destinadas a producciones intensivas (horticultura y fruticultura). El área total presenta un crecimiento de la población de 32\% (periodo 1996-2011) con una media anual de $2,1 \%$ con relación a 1996 . Este ritmo de crecimiento anual es muy superior al registrado a nivel país que se sitúa para el mismo periodo de $0,51 \%$ (INE, 2011b).

\subsection{Usos de suelo}

Se realizaron dos clasificaciones de uso de suelo, para 1993 y para 2013, mediante la lectura de imágenes satelitales (Jensen, 2000). Se utilizaron imágenes Landsat 5 y 7, disponibles en el Instituto Nacional de Pesquisas Espaciáis (INPE) ${ }^{6}$ e imágenes QuickBird, disponibles mediante la aplicación open layers over view del programa Quantum Gis 2.0. La clasificación se basó en las clases principales que propone la Ley de Ordenamiento Territorial (No 18.308)7 Urbano, Suburbano y Rural. Para poder tener una mayor heterogeneidad espacial se dividieron en subclases, diferenciándose 10 tipos de usos de suelo (tabla 1).

\footnotetext{
${ }^{5}$ Incremento de 49,282 a 65,053 habitantes (15,771 nuevos habitantes).

${ }^{6}$ Veáse INPE: http://www.inpe.br/

${ }^{7}$ Veáse http://www.parlamento.gub.uy/leyes/AccesoTextoLey.asp?Ley=18308\&Anchor=
} 
Tabla 1

Clasificación de las categorías y subcategorías del uso de suelo, periodo 1993-2013

\begin{tabular}{|c|c|}
\hline Categoría $^{8}$ & Subcategorias \\
\hline Urbano & $\begin{array}{l}\text { Consolidado } \\
\text { No consolidado }\end{array}$ \\
\hline Suburbano & $\begin{array}{l}\text { Aeropuertos } \\
\text { Centros logísticos-industriales } \\
\text { Zonas francas } \\
\text { Barrios privados } \\
\text { Complejos deportivos }\end{array}$ \\
\hline Rural & $\begin{array}{l}\text { Natural } \\
\text { Productivo } \\
\text { Abandonado }\end{array}$ \\
\hline
\end{tabular}

Fuente: elaboración propia con base en la Ley de Ordenamiento Territorial (No 18.308).

Estas categorías fueron digitalizadas a una escala de visualización 1:10.000. Finalmente, para la clasificación del 2013 se tomaron 15 puntos de muestreo (muestras de entrenamiento) para ajustar, verificar y validar la clasificación realizada en campo.

\subsection{Estratificación socioeconómica}

El concepto de clase es ante todo un concepto clasificatorio; es decir, intenta agrupar a distintos individuos a partir de un conjunto de rasgos comunes o criterios de unificación, vinculados con la posición social. Aquí naturalmente no se considera la discusión teórica de fondo, simplemente se busca plantear el concepto en el sentido de herramienta analítica. Se entiende que la ocupación constituye una dimensión central para observar la diferenciación de clases; sin embargo, no es posible contar con la misma para el estudio, una vez que el diseño y la elaboración de los censos están basados en estratos y no en clases sociales (Naciones Unidas, 2008). Por tanto, para aproximarse a la distribución espacial de las clases sociales se elaboró un índice de estratificación socioeconómica con base en los microdatos de los censos de población y vivienda de 1996 y 2011, realizados por el Instituto Nacional de Estadística (INE), en los cuales se identificaron a la educación, la vivienda-hogar y el trabajo como las dimensiones principales.

En estas dimensiones se seleccionaron distintas variables que permiten la comparación para ambos censos, ya que existió un cambio metodológico en la realización del censo del 2011 en relación con el censo de 1996,

${ }^{8}$ La definición de las categorías se encuentran detalladamente en la ley 18.308. 


\section{Tabla 2}

\section{Selección de las variables elaboradas por el censo, para la elaboración del índice de estratificación socioeconómica}

\begin{tabular}{|c|c|c|}
\hline Atributo de la variable & Variable & Dimensión \\
\hline $\begin{array}{l}\text { Pregrado } \\
\text { Primer grado } \\
\text { Segundo grado: primer ciclo } \\
\text { Segundo grado: segundo ciclo } \\
\text { Tercer grado }\end{array}$ & $\begin{array}{l}\text { Nivel educativo máximo } \\
\text { alcanzado9 }\end{array}$ & educación \\
\hline $\begin{array}{l}\text { Sí sabe } \\
\text { No sabe }\end{array}$ & Sabe leer y escribir & \\
\hline $\begin{array}{l}\text { Si planchada }{ }^{10} \\
\text { No planchada }\end{array}$ & $\begin{array}{l}\text { Material predominante en } \\
\text { techos }\end{array}$ & vivienda \\
\hline $\begin{array}{l}\text { Si mampostería }{ }^{11} \\
\text { No mampostería }\end{array}$ & $\begin{array}{l}\text { Material predominante en } \\
\text { paredes }\end{array}$ & \\
\hline $\begin{array}{l}\text { Si hormigón }{ }^{12} \\
\text { No hormigón }\end{array}$ & $\begin{array}{l}\text { Material predominante en } \\
\text { pisos }\end{array}$ & \\
\hline $\begin{array}{l}\text { Propietario } \\
\text { Arrendatario } \\
\text { Ocupante }\end{array}$ & Tenencia de la vivienda & \\
\hline $\begin{array}{l}\text { Número de vehículos } \\
\text { Número de computadoras } \\
\text { Número de máquina secadora } \\
\text { de ropa } \\
\text { Número de máquina lavadora } \\
\text { de ropa programada }\end{array}$ & $\begin{array}{l}\text { Confort y equipamiento del } \\
\text { hogar }\end{array}$ & hogar \\
\hline $\begin{array}{l}\text { Menores de } 12 \text { ańos } \\
\text { Ocupados } \\
\text { Desocupados } \\
\text { Inactivos }^{13}\end{array}$ & $\begin{array}{l}\text { Condición de actividad } \\
\text { económica }\end{array}$ & trabajo \\
\hline $\begin{array}{l}\text { Siempre residió } \\
\text { No siempre residió }\end{array}$ & Siempre residió en este lugar & \\
\hline
\end{tabular}

Fuente: INE (1996, 2011a).

\footnotetext{
${ }^{9}$ Clasificación basada en la guía de codificación de enseñanza del Centro Latinoamericano y Caribeño de Demografía (Celade).

${ }^{10}$ Se entiende como planchada al material de estructura rígida utilizando en el techo (compuesto por arena, portland y ladrillos) cuya función es proteger la vivienda del espacio exterior. Para el caso de una estructura no planchada se utilizan materiales livianos, quincha, barro o desecho.

${ }^{11}$ Se entiende como mampostería al material rígido con el que se construye la pared, cuya función es proteger la vivienda de resistencia, delimitación de los espacios y privacidad. Para el caso de una estructura sin mampostería se utilizan materiales livianos, quincha, barro o desecho.

${ }^{12}$ Se entiende como hormigón al material rígido con el que se construye el piso, cuya función es evitar que la vivienda se asiente en el suelo natural. Para el caso de la ausencia de hormigón se refiere a que la vivienda se asienta en suelo natural.

${ }^{13}$ Personas que no trabajan pero no buscan trabajo, ejemplo estudiantes mayores de 12 años.
} 
principalmente en la incorporación y eliminación de variables (INE, 2011a), se tuvieron que descartar las variables asociadas a la dimensión económica como la mediación de los ingresos de las personas (tabla 2). La unidad espacial considerada durante este estudio fue la utilizada por el INE, denominada Segmento Censal, dividida en función de la densidad de población y el amanzanamiento.

Posteriormente, se procedió a la realización del índice de estratificación socioeconómica en función de las carencias básicas que presenta la población para este conjunto de variables. En este sentido, se le asignó mayor valor a: i) los niveles más bajos de educación; ii) si los materiales constitutivos de la vivienda están basados en elementos precarios; iii) si existe ausencia de los elementos de confort, y iv) si la condición de la actividad económica es desfavorable (trabajo). Finalmente, para poder visualizar de forma más clara los resultados, se elaboraron tres categorías cualitativas (bajas, medias y altas), con base en la desviación estándar.

\section{Resultados y discusión}

La elaboración del SIG, mediante la realización de la clasificación de usos de suelo y el índice de estratificación socioeconómica, permitió aproximarse a comprender la dinámica territorial, registrando variaciones significativas en los últimos 20 años. Para una mejor comprensión de los resultados se muestran, por un lado, los referentes a los usos de suelo y, por otro, los resultados de estratificación socioeconómica.

\subsection{Usos de suelo}

América Latina sufre transformaciones en el cambio de uso de suelo de forma acelerada durante las últimas décadas (Turner et al., 1995; Lambin $y$ Geist, 2006; Baldi y Paruelo, 2008), una de ellas es el cambio de uso rural a urbano; si bien es la transformación que abarca menos superficie, a diferencia del avance de la frontera agrícola, es la más intensa ya que existe una modificación en toda su función (Lambin y Geist, 2006). Un indicador indirecto de estos cambios es el crecimiento de la población urbana en función de la disminución de la población rural: se observa un incremento de 8,9\%, para el periodo 1990-2010, proceso que se ve reflejado en el crecimiento de todas las regiones metropolitanas de América Latina (Cepal, 2012).

Uruguay presenta un comportamiento diferencial, por un lado, existe un incremento de su población urbana de $13,7 \%$ para el mismo periodo y, por otro lado, la región metropolitana de la ciudad de Montevideo registra un decrecimiento de $0,4 \%$ de su población, por lo que, según 
informes de AMM (2007), Cepal (2012) e INE (2011b) es la única región metropolitana de América Latina con decrecimiento. Para el área de estudio, el uso del suelo muestra un comportamiento diferente al resto de la región metropolitana de Montevideo (mapa 2), donde se observa un crecimiento del uso de suelo urbano y suburbano, principalmente, debido a la instalación de industrias y empresas logísticas junto con la construcción de barrios privados. Las industrias y empresas logísticas se concentran sobre el eje vial No 101, de tal forma que suman más de diez los nuevos emprendimientos instalados. Se destaca la presencia de una nueva zona franca denominada "Parque de las Ciencias", la cual se caracteriza por la concentración de multinacionales farmacéuticas.

Los barrios privados se construyeron a partir de 1996 y actualmente son cerca de siete. Esta cifra en el contexto latinoamericano puede parecer pequeña, pero para Uruguay es significativa, tomando en cuenta que hay un debate político sobre la instalación de los barrios privados, principalmente porque Montevideo prohíbe este tipo de emprendimiento. También se observa, en menor medida, la construcción de campos deportivos asociados a clubes de fútbol y a centros de enseñanza privada. Cabe destacar que recientemente se comenzó con una megaobra de infraestructura para la dimensiones del Uruguay, en las confluencias del eje vial No 8 y No 102, el estadio de fútbol del Club Atlético Peñarol, que albergará a unas 40 mil personas.

Otro elemento a resaltar es el aumento de los asentamientos irregulares que han crecido de forma importante pasando de 19 a 29 para la totalidad del área.

Con respecto al uso rural, creció el área abandonada 4,0\%, en la influencia del nuevo anillo perimetral y la superficie productiva disminuyó $11,0 \%$, resistiendo pequeñas unidades productivas destinadas a la producción hortícola.

\subsection{Estratificación socioeconómica}

Los procesos de fracturación, estratificación y diferenciación social que ocurren en las ciudades latinoamericanas son fenómenos recurrentes que afectan la estructura y morfología de las ciudades (Ferreira, 2003; De Mattos, 2004; Harvey, 2007; Ziccardi, 2008). En el AMM este proceso se vio agudizado con la profundización del modelo neoliberal durante la década de 1990 y comienzos del 2000 (Boado y Fernández, 2005; Serna, 2010). Esta situación, se materializa con el crecimiento del número de asentamientos irregulares en la ciudad, proceso por el cual se favoreció y profundizó la segregación territorial en el área (Veiga, 2009). 


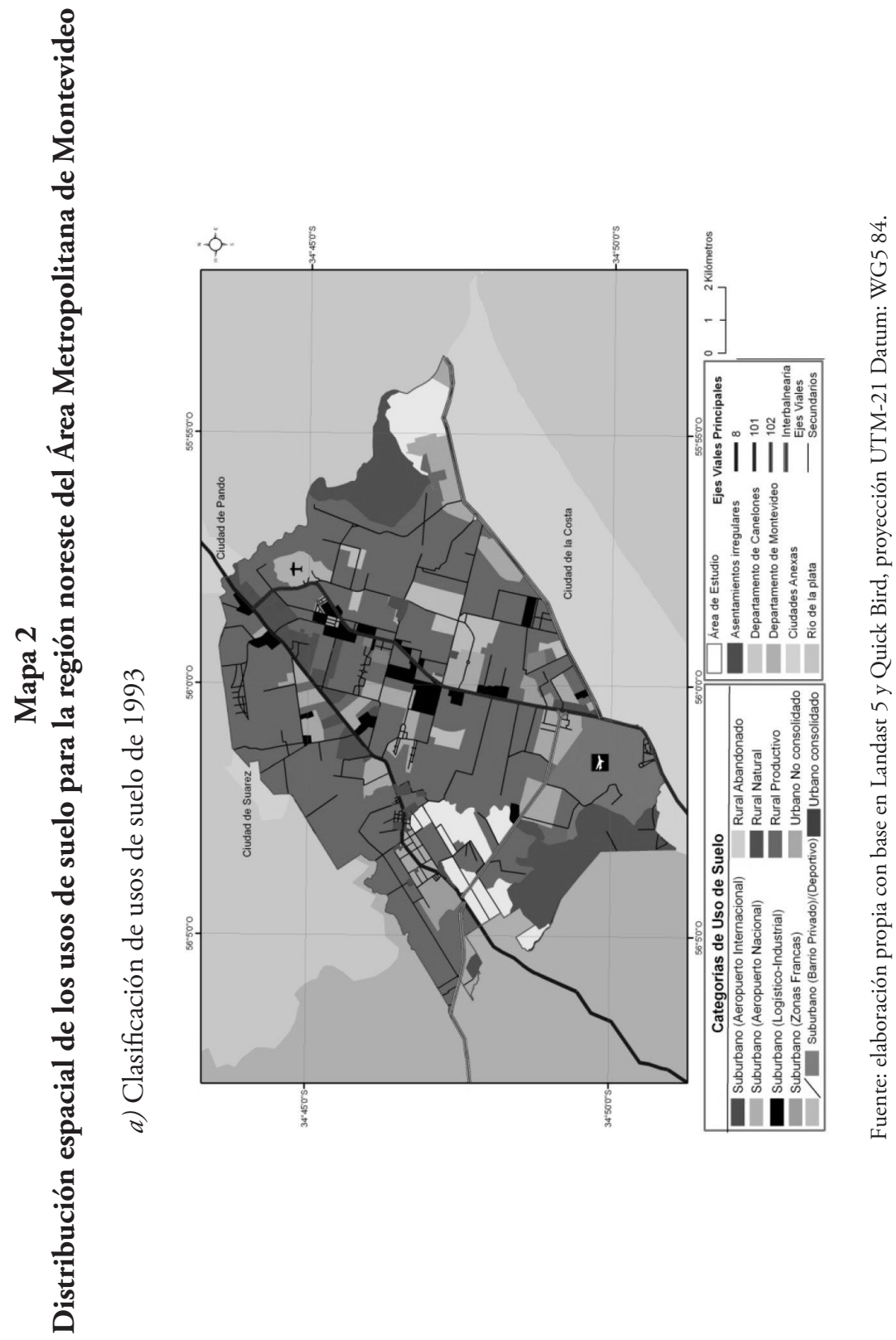




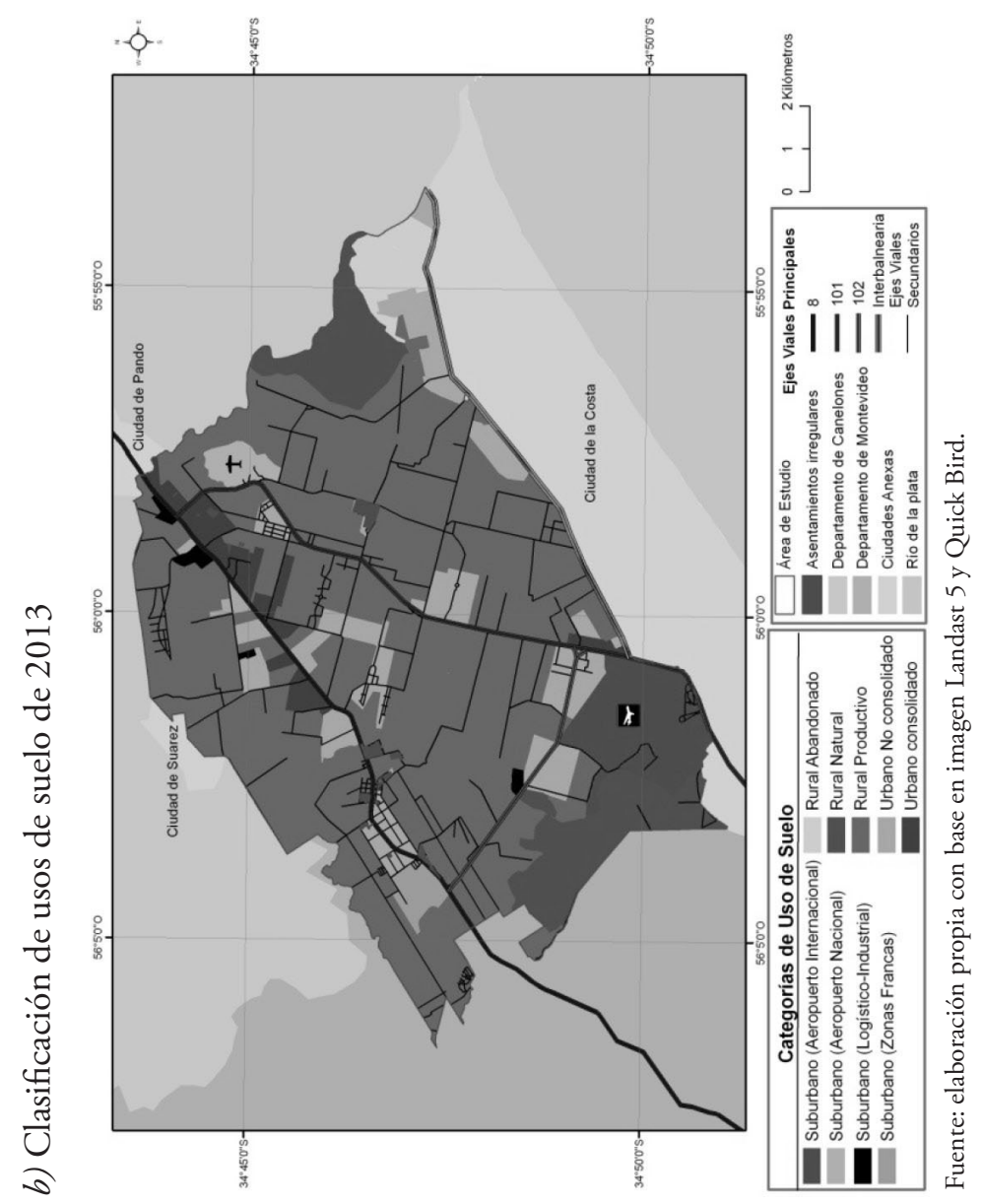


En relación al área de estudio, se aprecia una clara diferenciación territorial de las distintas clases sociales, tanto para 1996 como para 2011 (mapa 3). Como se observa para 1996, la clase social alta se distribuye de forma fragmentada, representando $6,0 \%$ del total de la superficie, mientras que la clase media ocupaba mayor superficie, ubicándose con mayor presencia en la zona urbana en 56,5\% del total. Finalmente, la clase baja localizada en mayor medida en las zonas rurales registró 37,5\% del total. En relación al 2011, la clase alta se consolida y se concentra al sur del eje vial No 101 con la materialidad de los barrios privados ocupando 3,9\% del total de la superficie, mientras que la clase media ocupa $46,3 \%$ del total y, finalmente, la clase baja es la que ocupa la mayor superficie con $49,8 \%$ del total.

Con respecto a la variación de las clases en relación al número de habitantes, para 1996, 12,7\% de los habitantes se agrupa en la clase alta, $70,9 \%$ se concentra en la clase media y $16,4 \%$, en la clase baja. Para el 2011, la clase alta registra 2,4\%, posteriormente, la clase media $47,9 \% \mathrm{y}$, finalmente, la clase baja con 49,7 por ciento.

La tendencia de los comportamientos tanto en superficie como en número de habitantes es similar, existiendo dos grandes resultados, por un lado, el crecimiento de la clase baja, del orden de 33\% y, por otra parte, una disminución de $23 \%$ de la clase media, mientras que la clase alta registra una leve disminución.

El crecimiento de la clase baja puede estar asociado a un deterioro de los ingresos de los hogares principalmente rurales, debido a la baja rentabilidad de las unidades productivas (UP), resultado de la ausencia de políticas del Estado hacia este sector, que genera un desplazamiento de los productores. Según el último censo agropecuario, la producción hortícola asociada a cultivos de huerta se ha reducido en $55 \%$, siendo las UP más pequeñas ( 0 a 0,5 hectáreas), predominantes en el área de estudio, las que han desaparecido en casi su totalidad en 96\% (MGAP, 2014).

Esta desaparición se materializa en el abandono y la venta de la unidad productiva, lo que abre la posibilidad de compra de tierras de bajo valor. Este mecanismo puede ser una atracción para la clase baja urbana que necesita de tierras baratas para instalarse. Con relación a la disminución de la clase media podría deberse a dos posibilidades: una asociada a la migración de esta clase a otras zonas, o el empobrecimiento de la propia población, aunque ésta es más difícil de afirmar debido a la carencia de algunos datos como los propios ingresos de las personas. Finalmente, existe una nueva distribución espacial de la clase alta, mayormente concentrada, la cual está asociada a la instalación de los barrios privados, un tipo de fraccionamiento inexistente hasta 1997. 


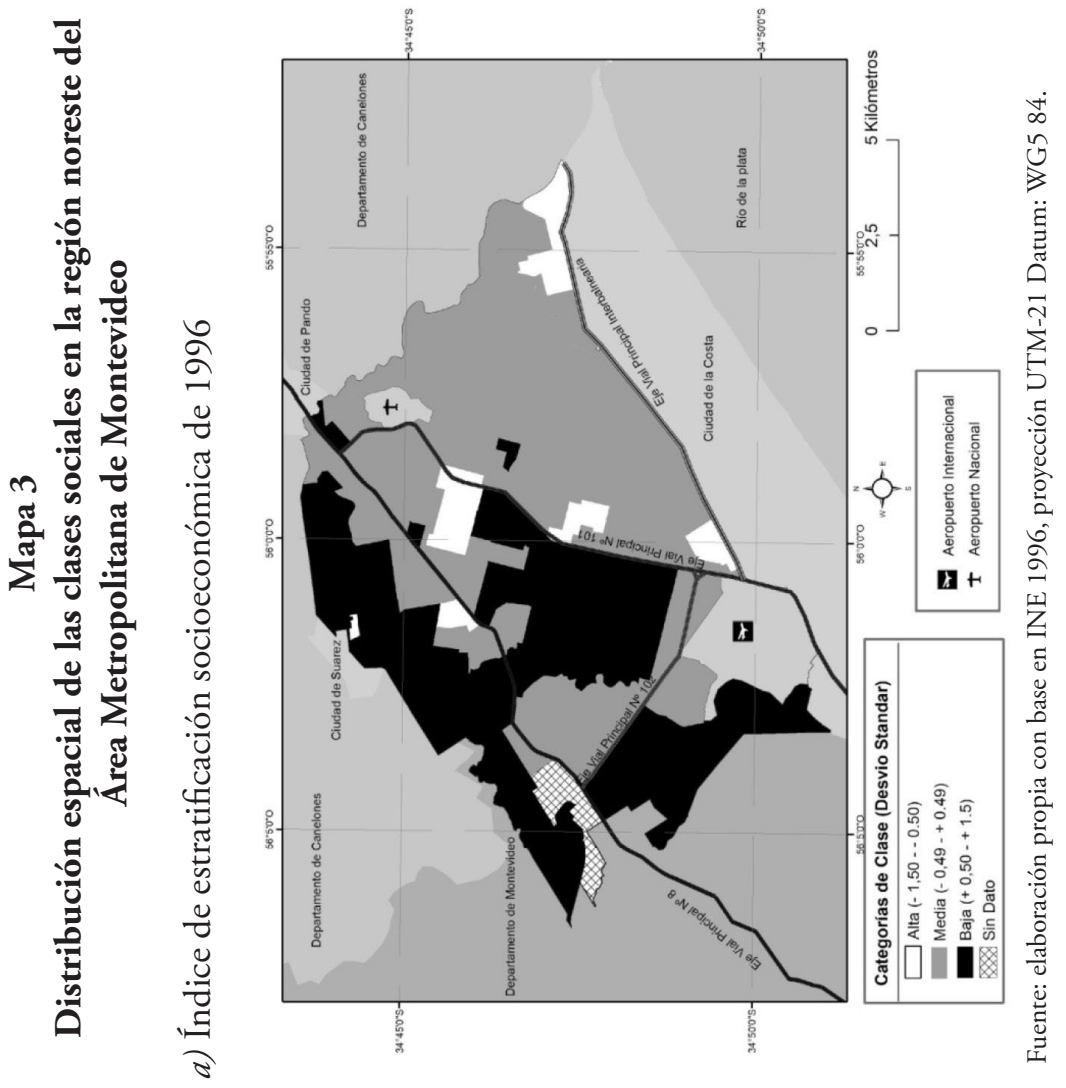




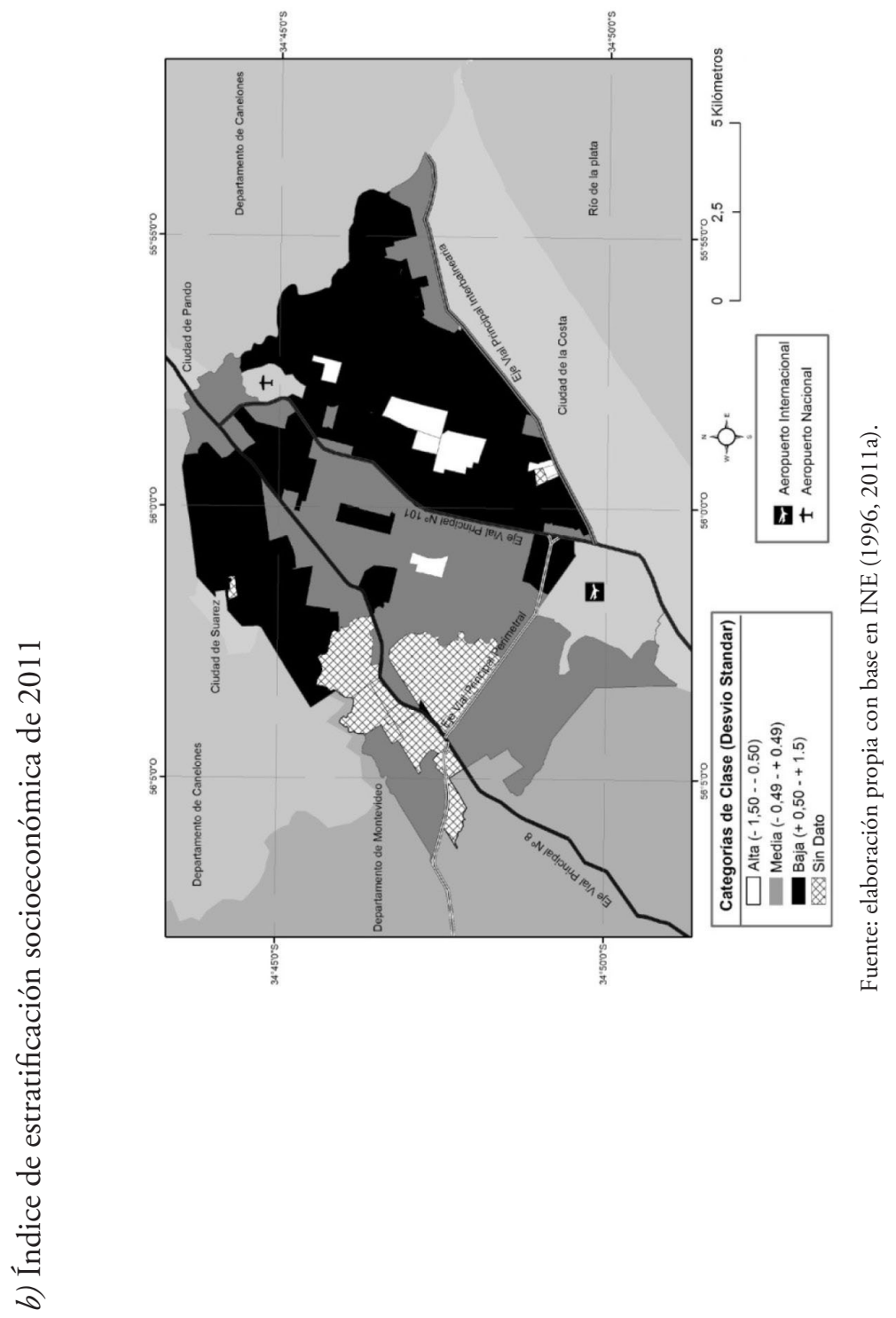


En definitiva, se percibe cómo las clases sociales se distribuyen en el territorio, presentando una reconfiguración de la apropiación territorial, así como una diferente concentración que genera una mayor segregación visible en el paisaje.

A través de las dos categorías analíticas se evidenció que durante las últimas dos décadas se presentaron cambios significativos en varios ámbitos. Entre ellos se encuentra el uso de suelo, por medio de la apropiación de distintos estratos socioeconómicos que se concentran y constituyen distintos territorios, muchas veces segregados. Otra transformación se observa en la cantidad de inversiones públicas, principalmente relacionados con la conectividad, como del sector privado a través de empresas transnacionales, logísticas y estilo de vida/barrios privados. En general estos cambios ocurren de una manera interconectada y muchas veces en una dependencia mutua.

\section{Conclusiones}

Los resultados muestran que las transformaciones territoriales ocurren de forma acelerada y acumulativa, diferenciándose del comportamiento general del AMM. En el marco de este análisis se ve cómo la segregación territorial en el área está determinada, entre otros factores, por la forma de apropiación de uso del suelo por diferentes clases sociales. Además indirectamente muestra que el Estado tiene un rol protagonista en las diversas transformaciones y muchas veces actúa en la configuración de los territorios estratégicos.

Este protagonismo se expresa a través de la creación de normativa legal, materializada en la creación de servicios e infraestructuras, las que favorecen el aumento de la reproducción del capital. El papel que desempeña la normativa es importante, tanto a escala nacional como departamental. A nivel nacional, a partir de la llamada Ley de Zonas Francas (No 15.921), la Ley de Protección y Promoción de Inversiones (No 16.906), la Ley de Parques Industriales (No 17.547) y la Ley de Inversión PúblicoPrivada (No 18.786) se genera el marco necesario para la exoneración de impuestos y el estímulo para la instalación de capital, principalmente, de origen multinacional.

Por otro lado, a nivel departamental existen las directrices y ordenanzas de la Ley de Ordenamiento Territorial que han favorecido la creación de territorios con mayores o menores beneficios para su localización. Reflejo de esta situación son el crecimiento de las industrias en el eje vial No 101 y la creación de los barrios privados, demostrando como el propio Estado es un actor clave para generar las condiciones de la transformación territorial. 
En relación a las infraestructuras, la creación del Anillo Colector Vial Perimetral genera una disminución de costos del transporte de mercancías lo que también favorece la instalación del capital, sumado a la cercanía de centros de conectividad internacional (aeropuerto). En este sentido, el área se configura como parte de la lógica del territorio estratégico, donde la reproducción del capital traspasa las fronteras estatales, generando embates y contradicciones legales. Un ejemplo de ello, es el cambio de uso de suelo, que termina siendo una herramienta política. Entre las consecuencias notables que trae consigo se encuentra: el desplazamiento de pobladores rurales, la valorización y especulación de la tierra, el aumento de impuestos y la necesidad de infraestructura. Aun dentro de esta lógica, la apropiación del suelo por distintas clases sociales cambia la configuración territorial, ampliando las diferencias y la segregación entre las clases.

De esta manera, las transformaciones que están ocurriendo en el área de estudio se pueden interpretar bajo dos hipótesis explicativas: la primera, que predomina en los tomadores de decisiones, se refiere a la instalación de los enclaves de capital, como el ejemplo de las zonas francas y los barrios privados que pueden actuar como motor de atracción de mano de obra y darle dinamismo y crecimiento en términos de ingreso económico a la zona. La segunda, utilizada por algunos autores, entre ellos Falero (2011), que demuestran cómo los enclaves de capital requieren mano de obra calificada que generalmente no está presente en la zona, lo cual ocasiona un aumento en la segregación territorial. Esta hipótesis converge con nuestros resultados, ya que a lo largo del artículo se visualizó que los procesos de empobrecimiento y segregación territorial han crecido en la región noreste del AMM, a pesar de las inversiones hechas que favorecen a algunos sectores específicos en detrimento de la mayoría.

Es importante destacar la necesidad de un ordenamiento territorial más justo, ambientalmente sustentable y participativo, donde se integren los intereses de todos los actores en beneficio de una sociedad metropolitana que priorice la cohesión social en detrimento de los aumentos de segregación y marginalización de la población. La integración y flexibilidad en la normativa en el AMM debería ser de interés general por encima del interés particular, apegarse a lo que establece la Ley de Ordenamiento Territorial (No 18.308) principalmente en lo correspondiente a los instrumentos interdepartamentales y especiales, referidos en el artículo 8. Un ejemplo de ello es incongruencia departamental que existió en la autorización de los barrios privados para el caso de Canelones y la prohibición para Montevideo.

Lejos de finalizar las discusiones, los resultados abren nuevos cuestionamientos que convergen con la necesidad de seguir analizando los 
procesos y fenómenos que ocurren en el área, que actualmente se presenta como un territorio estratégico que afecta y profundiza la segregación territorial

\section{Fuentes consultadas}

Álvarez-Rivadulla, María José (2007), "Golden ghettos: gated communities and class residential segregation in Montevideo, Uruguay", Environment and Planning A, 39 (1), Sage Publications, pp. 47-63.

AMM (Agenda Metropolitana de Montevideo) (2007), Libro blanco del área metropolitana, (Canelones, Montevideo, San José), Agenda Metropolitana de Montevideo, Montevideo.

Araújo, Federico Guilherme Bandeira de y Rogério Haesbaert (2009), "Territórios e identidades: questóes e olhares contemporâneos", Trabalho, Educação e Saúde, 7 (3), Fundação Oswaldo Cruz, Escola Politécnica de Saúde Joaquim Venâncio, Rio de Janeiro, pp. 629-633.

Baldi, German y José Paruelo (2008), "Land-use and land cover dynamics in South American temperate grasslands", Ecology and Society, 13 (2): 6, <http://www.ecologyandsociety.org/vol13/iss2/art6/>, 1 de octubre de 2013.

Boado, Marcelo y Tabaré Fernández (2005), “Una mirada a la crisis, las clases y la pobreza en el Uruguay 1998-2004”, ponencia presentada en el Seminario México-Uruguay sobre Medición de la Pobreza y Políticas Sociales, 27 y 28 de julio 2005, Montevideo.

Bourdieu, Pierre (1998), A miséria do mundo, Vozes, Petrópolis.

Cepal (Comisión Económica para América Latina y el Caribe) (2012), Anuario Estadístico de América Latina y el Caribe 2012, Comisión Económica para América Latina y el Caribe, <http://www. eclac.org/cgibin/getProd.asp?xml=/publicaciones/xml/2/48862/ P48862.xml\&xsl=/publicaciones/ficha.xsl\&base=/publicaciones/ top_publicaciones.xsl>, 10 de diciembre de 2013.

De Mattos, Carlos (2004), "Redes, Nodos e Cidades: transformação da metrópole latino- americana”, en Luiz Cesar Queiroz Ribeiro, (coord.), Metrópoles: entre a coesão e a fragmentação, a cooperação 
e o conflito, Editora Fundação Perseu Abramo, Rio de Janeiro, pp. 157-196.

Falero, Alfredo, Marcelo Pérez, Mauricio Ceroni, Aline da Fonseca y Alicia Rodríguez (2013), "Cambios y disputas territoriales: el caso de la región metropolitana noreste de Montevideo", Revista Contrapunto, 3 (1), Universidad de la República, Montevideo, pp. 33-45.

Falero, Alfredo (2011), Los enclaves informacionales de la periferia capitalista: el caso de Zonamérica en Uruguay. Un enfoque desde la sociología, Comisión Sectorial de Investigación Científica (CSIC), Montevideo.

Ferreira Cardoso da Silva, Alexsandro (2003), "O parcelamento do solo e a formação de espaços de pobreza em Natal-RN”, Scripta Nova: revista electrónica de Geografía y Ciencias Sociales, VII, 146 (130), Universidad de Barcelona, Barcelona.

García-Gómez, Carmen y Tonatiuh Claudio Ruiz-Salazar (2011), "La segregación territorial y el rezago en el sur de la ciudad de Mérida, como el resultado del crecimiento urbano descontrolado", Quivera, 13 (1), Universidad Autónoma del Estado de México, Toluca, pp. 122-138.

Harvey, David (2007), Social justice and the city, Johns Hopkins University, Baltimore.

Harvey, David (2001), "Possible urban worlds", Lotus International, 110 (1), La Haya, pp. 18-22.

Hernández, Facundo (2009), "Urbanizaciones privadas en América Latina, los 'guetos' del siglo XXI. El caso del crecimiento de countries y barrios privados en la costa atlántica Argentina”, ponencia presentada en el EGAL. Caminando en una América Latina en Transformación, 3-7 de abril de 2009, Universidad de la República, Montevideo.

INE (Instituto Nacional de Estadística) (2011a), Consideraciones metodológicas y conceptuales sobre los cuestionarios de Población, Hogares y Viviendas de los Censos 2011, Instituto Nacional de Estadística, Montevideo, <http://www.ine.gub.uy/censos2011/ resultadosfinales/consideraciones.pdf>, 10 de agosto de 2013. 
INE (Instituto Nacional de Estadística) (2011b), Resultados del Censo de Población 2011: población, crecimiento y estructura por sexo y edad, Instituto Nacional de Estadística, Montevideo, <http:// www.ine.gub.uy/censos2011/resultadosfinales/analisispais.pdf>, 10 de agosto de 2013.

INE (Instituto Nacional de Estadística) (1996), Resultados del Censo de Población 1996: VII Censo General de Población y V de Viviendas, Instituto Nacional de Estadística, Montevideo, <http:// www3.ine.gub.uy:82/anda4/index.php/catalog/45>, 3 de julio de 2013.

Jensen, Jonh (2000), Remote sensing of the environment: an earth resource perspective, Upper Saddle River-Prentice Hall, New Jersey.

Lambin, Eric y Helmut Geist (2006), Land use and land cover change: local processes and global impacts, Springer, Berlín.

Landsat 5 (2013), sensor multiespectral, bandas 3,4,5, Instituto Nacional de Pesquisas Espaciáis, Brasil, 20 de mayo de 2013.

Lombardi, Mario y Federico Bervejillo (1999), “Globalización, integración y expansión metropolitana en Montevideo”, ponencia presentada en el V Seminario Internacional de la RII, 21-24 de septiembre, Toluca.

Martínez, Edgardo (2011), "Reportaje de ciudades, transformaciones urbanas y sus pobladores en Montevideo metropolitano", Cuaderno urbano, 11 (11), Universidad Nacional del Nordeste, Corrientes, pp. 150-174.

Martins, José (1997), Exclusão e a nova desigualdade, Paulus Editora, São Paulo.

Mendy, Mariana y Victoria Gónzalez (2010), "El territorio como ámbito de expresión de las desigualdades sociales, clases sociales y políticas en el desarrollo", <http://www.pim.edu.uy/files/2013/04/ El-Territorio-como-\%C3\%A1 mbito-de-expresi\%C3\%B3n-delas-desigualdades-sociales.pdf>, 10 de septiembre de 2013. 
MGAP (Ministerio de Ganadería Agricultura y Pesca) (2014), Censo General Agropecuario 2011: Resultados Definitivos, Ministerio de Ganadería Agricultura y Pesca, Montevideo.

Naciones Unidas (2008), Principios y Recomendaciones para los Censos de Población y Vivienda, Revisión 2. Comisión de Estadística, $<$ http://unstats.un.org/unsd/publication/SeriesM/Seriesm_67 rev2s.pdf>, 1 de octubre de 2013.

Oliveira, Francisco (1998), Os direitos do anti valor: a economia politica da hegemonia imperfeita, Editora Vozes, Petrópolis.

Pérez-Campuzano, Enrique y Clemencia Santos-Cerquera (2011), "Diferenciación socioespacial en la Zona Metropolitana de la Ciudad de México", Investigaciones Geográficas, núm. 74, Instituto de Geografía, Universidad Nacional Autónoma de México, México, pp. 92-106.

Poder Legislativo, Ley No 18.786, 19 de julio de 2011, Senado y la Cámara de Representantes, Uruguay, <http://www.parlamento.gub. uy/leyes/Acceso TextoLey.asp?Ley=18786\&Anchor=>, 19 de octubre de 2013.

Poder Legislativo, Ley No 18.308, 18 de junio de 2008, Senado y la Cámara de Representantes, Uruguay, <http://www.parlamento. gub.uy/leyes/Acceso TextoLey.asp?Ley=18308\&Anchor=>, 29 de agosto de 2013.

Poder Legislativo, Ley No 17.547, 22 de agosto de 2002, Senado y la Cámara de Representantes, Uruguay, <http://www.parlamento. gub.uy/leyes/Acceso TextoLey.asp?Ley=17547\&Anchor=>, 29 de octubre de 2013.

Poder Legislativo, Ley No 16.906, 7 de enero de 1998, Senado y la Cámara de Representantes, Uruguay, <http://www.parlamento.gub. uy/leyes/Acceso TextoLey.asp?Ley=16906\&Anchor=>,15 de octubre de 2013.

Poder Legislativo, Ley No 15.921, 17 de diciembre de 1987, Senado y Cámara de Representantes, Uruguay, <http://www.parlamento. gub.uy/leyes/AccesoTextoLey.asp?Ley=15921\&Anchor=>, 15 de octubre de 2013. 
QGIS (Quantum GIS) (2013), aplicación open layers over view-Googlestreets, versión 2.0, España.

Quick Bird (2013), sensor multiespectral, bandas 3,4,5, Digital Globe, Estados Unidos, 23 de noviembre de 2013.

Sassen, Saskia (2007), Una sociología de la globalización, Katz Editores, Buenos Aires.

Saquet, Marcos Aurelio (2007), Abordagens e concepçóes sobre o território, Expressão Popular, São Paulo.

Schelotto, Salvador (2008), “La ciudad de Montevideo: ¿Una metrópoli policéntrica?”, Centro-h, núm. 2, Organización Latinoamericana y del Caribe de Centros Históricos, Quito, pp. 37-46.

Serna, Miguel (2010), Pobreza y (des) igualdad en Uruguay: una relación en debate, Universidad de la República-Facultad de Ciencias SocialesDepartamento de Sociología-Asdi-CLACSO, Montevideo.

Turner, Billie Lie, David Skole, S. Sanderson, G. Fisher, L.O. Fresco, Rik Leemans (1995), "Land-use and land-cover change. Science/ Research Plan”, Global Change Report, Royal Swedish Academy of Sciences, Estocolmo.

Veiga, Danilo (2013), “Estratificación social, desigualdades y segregación urbana en Montevideo", ponencia presentada en el II Congreso Uruguayo de Sociología, 10-12 de julio de 2013, Universidad de la República, Montevideo.

Veiga, Danilo (2009), “Desigualdades sociales y fragmentación territorial en el área metropolitana de Montevideo", Revista Lider, 15 (15), Universidad de los Lagos, Chile, pp. 127-153.

Veiga, Danilo y Ana Laura Rivoir (2001), Desigualdad social y segregación en Montevideo, Universidad de la República, Montevideo.

Ziccardi, Alicia (2008), Procesos de urbanización de la pobreza y nuevas formas de exclusión social. Retos de las politicas sociales de las ciudades latinoamericanas del siglo XXI, Siglo del Hombre Editores, CLACSO, Bogotá. 
Recibido: 21 de abril de 2014. Corregido: 13 de enero de 2015. Aceptado: 22 de marzo de 2015.

Mauricio Ceroni. Estudiante de doctorado en geografía por la Universidad Nacional Autónoma de México, magister en ciencias ambientales por la Universidad de la República, Uruguay. Actualmente es docentecolaborador del Laboratorio de Desarrollo Sustentable y Gestión Ambiental del Territorio, Instituto de Ecología y Ciencias Ambientales, Facultad de Ciencias, Universidad de la República, Uruguay. Entre sus principales líneas de investigación destacan el análisis y sus impactos de las transformaciones territoriales que ocurren en la cobertura terrestre desde una dimensión biofísica y socioeconómica, con énfasis en la dinámica urbana-rural. Entre sus últimas publicaciones, en coautoría, destacan: "IIRSA: otro paso en la explotación de los pueblos y territorios sudamericanos", REDES-AT, 1 (1), Amigos de la Tierra, Montevideo, pp. 1-69 (2007); "Análise do balanço hídrico superficial e avaliação da disponibilidade potencial da água para o rodeio do gado no Uruguai entre $1980 \mathrm{e}$ 2006", Revista Acadêmica, (8) 2, Pontificia Universidade Católica do Paraná, Curitiba, pp. 171-181 (2010); "Cambios y disputas territoriales: el caso de la región metropolitana noreste de Montevideo", Revista Contrapunto, 3 (1), Universidad de la República, Montevideo, pp. 33-45 (2013).

Aline da Fonseca. Magister en educación, cultura y comunicación en periferias urbanas de Universidad do Estado do Rio de Janeiro, Brasil. Actualmente es docente del Laboratorio de Desarrollo Sustentable y Gestión Ambiental del Territorio, Instituto de Ecología y Ciencias Ambientales, Facultad de Ciencias y del Programa Integral Metropolitano de la Universidad de la República, Uruguay. Su área de trabajo actual se centra en análisis de estudios urbanos con énfasis en conflictos territoriales y en la extensión universitaria. Entre sus últimas publicaciones, en coautoría, destacan; "Cambios y disputas territoriales: el caso de la región metropolitana noreste de Montevideo", Revista Contrapunto, 3 (1), Universidad de la República, Montevideo, pp. 33-45 (2013); "Habitar las diferencias en la construcción de 'nosotros': integración barrial e interdisciplina", ponencia presentada en el Extenso 2013, 6-9 de noviembre, Montevideo; "En diálogo con las prácticas territoriales: la experiencia del Seminario de Prácticas Integrales", ponencia presentada en el Extenso 2013, 6-9 de noviembre, Montevideo.

Feline Schön. Master en geografías de la globalización por la Goethe Universität, Frankfurt/Main, Alemania. Actualmente es docente del La- 
boratorio de Desarrollo Sustentable y Gestión Ambiental del Territorio, Instituto de Ecología y Ciencias Ambientales, Facultad de Ciencias, Universidad de la República, Uruguay. Su área de trabajo actual se centra en análisis de estudios urbanos con énfasis en conflictos territoriales y movimientos sociales. Entre sus últimas publicaciones, en coautoría, destaca: "Megaminería y fractura hidráulica: potenciales impactos ambientales y para el derecho humano al agua", REDES-AT, Amigos de la Tierra, Montevideo, pp. 4-40 (2014).

Ana Domínguez. Doctora en geografía de estudios de América Latina, Universidad de Toulouse II (Le Mirail), Francia. Actualmente es docente titular del Laboratorio de Desarrollo Sustentable y Gestión Ambiental del Territorio, Instituto de Ecología y Ciencias Ambientales, Facultad de Ciencias, Universidad de la República, Montevideo, y docente titular del Instituto de Profesores Artigas, Uruguay. Su área de trabajo actual se centra en la evaluación de las políticas de desarrollo rural en América Latina, junto con análisis de las políticas de gestión de los recursos hídricos en Uruguay. Entre sus últimas publicaciones, en coautoría, destacan: "Hacia un escenario de gestión sustentable del territorio por cuencas hidrográficas en el contexto post neoliberal en el Uruguay", Revista Cientifica Monfragüe, IV (1), Universidad de Extremadura, Extremadura, pp. 104-123 (2015); "Concentración, extranjerización y exclusión agraria en el Uruguay rural contemporáneo", Asociación Latinoamericana de Sociología Rural, 6 (1), Alasru, Montevideo, pp. 209-242 (2012); "La intensificación del uso agrícola del suelo en el litoral oeste del Uruguay en la última década", Pampa, 7 (7), Universidad Nacional del Litoral, Santa Fe, Argentina, pp. 143-158 (2011). 